\title{
PHOTONIC BANDGAP STRUCTURE BASED ACCELERATING CELL
}

\author{
M.A.Shapiro, W.J.Brown, and R.J.Temkin, Plasma Science and Fusion Center, \\ Massachusetts Institute of Technology, Cambridge MA 02139
}

\section{Abstract}

We present detailed calculations of photonic bandgap (PBG) accelerating cavities including estimation of the important effects of an input coupler. The PBG structures consist of a triangular lattice of metal rods containing a single defect on axis. The operating frequency is selected to be $17.1 \mathrm{GHz}$. The accelerating mode is a quasi- $\mathrm{TM}_{010}$ mode localized near the defect. We analyzed the excitation of the cell using a rectangular waveguide with aperture coupling. Both the design of the PBG cell and the design of the input coupling were varied in order to optimize the design. Results are compared with a conventional $\mathrm{TM}_{010}$ cavity design. Ohmic loss is found to be comparable for the different cavity designs. The results indicate that the PBG cavity can have the following advantages relative to a conventional cavity: rarified spectrum of modes; oversized dimensions; and simplified input coupling with no frequency shift.

\section{INTRODUCTION}

Photonic bandgap structures (photonic crystals) [1] have recently found a number of applications at the frequencies from microwave to optical [2]. A 2-D photonic bandgap structure based accelerating cell was proposed by S.Schulz et al. at PAC'93 [3]. The cavities made of metal and dielectric rods with a single defect were analyzed, and the defect mode was observed in a cold test in X-band [4-6].

PBG based accelerating cells may be attractive at higher frequencies (17 GHz and up to $90 \mathrm{GHz}$ ) since the cavity is oversized. Therefore, the following features can be employed: (a) oversized quasi-optical waveguides can be utilized because conventional rectangular waveguides have large Ohmic losses at these frequencies; (b) the operating mode is localized near the defect whereas higher order, higher frequency modes fill the entire volume of the cavity and, therefore, they can be damped or removed.

The MIT experimental facility operating at $17 \mathrm{GHz}$ includes a 1-1/2-cell photoinjector RF gun [7], the Haimson Research Corporation (HRC) $36 \mathrm{MeV}$ linear accelerator [8] and HRC $20 \mathrm{MW}$ relativistic klystron. This report is devoted to the design a $17 \mathrm{GHz}$ PBG structure based accelerating cell. This cell may be accommodated and experimentally investigated at MIT.
The design we present includes calculations of the frequency, transverse field distribution, and Ohmic Qfactor of the defect mode as well as analysis of coupling into the defect mode using a rectangular waveguide.

\section{PBG CAVITY MODE}

\subsection{Frequency and Field Distribution}

We use a triangular lattice of metal rods with a defect to form a PBG cavity (Fig1). Since a finite number of circular rows of rods is required to localize the defect mode, we utilize only 3 rows of rods. The rods are of a finite length and placed between two metal planes parallel to the plane of the drawing (Fig.1). The operating mode is a quasi- $\mathrm{TM}_{010}$ mode. Therefore, the electric field is parallel to the rods, and the magnetic field is in the plane of the drawing.

We use SUPERFISH code [9] to examine two PBG cavities. The parameters of the cavities are shown in Table 1. Figure 1 plots the lines of constant electric field in Cavity \#1. It is seen that the mode is localized near the defect and slightly penetrates to the space between the $2^{\text {nd }}$ and $3^{\text {rd }}$ rows. The results of the resonance frequency calculation are shown in Table 1.

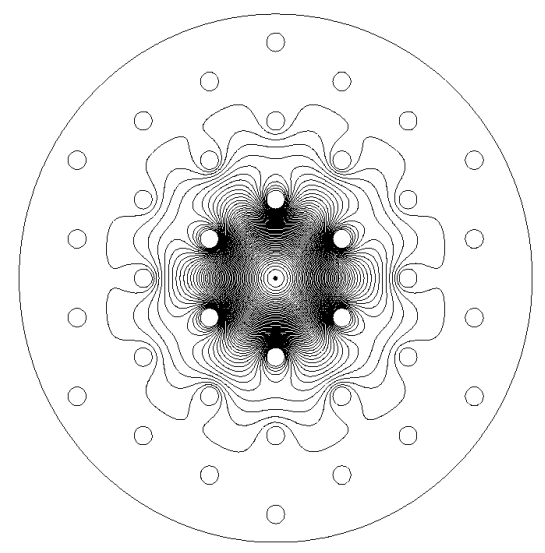

Fig.1. Electric field in Cavity \#1.

\subsection{Ohmic Losses and Shunt Impedance}

The Ohmic Q-factor of the cavity is expressed as follows:

$$
Q_{o h m}=d_{s k}^{-1}\left(1 / R_{\text {eff }}+1 / L_{\|}\right)^{-1}
$$

where the skin-layer depth $d_{s k}=0.5 \mu \mathrm{m}$ at $17 \mathrm{GHz}, L_{\|}$is the axial length, and an effective radius of the mode, $R_{\text {eff }}$, is 


$$
R_{\text {eff }}=2 \int_{S_{\perp}} H^{2} d s_{\perp}\left(\oint_{l_{i}} H^{2} d l\right)^{-1}
$$

where the magnetic field $H$ is integrated over the cavity cross-section $S_{\perp}$ and over all contours $l_{i}$ of the rods.

SUPERFISH output gives the stored energy per unit of axial length:

$$
W=\frac{\mu_{0}}{2} \int_{S_{\perp}} H^{2} d s_{\perp}
$$

where $\mu_{0}=4 \pi \cdot 10^{-7} \mathrm{H} / \mathrm{m}$. The contour integral can be determined using the SUPERFISH data. Note that $R_{\text {eff }}$ is equal to the cavity radius $R$ for a conventional pill-box cavity. A specific shunt impedance is expressed as follows:

$$
r_{c y}=\frac{E_{\max }^{2}}{\omega W} Q_{o h m}
$$

\begin{tabular}{|c|c|c|c|c|}
\hline & \multicolumn{2}{|c|}{ PBG Cavity \#1 } & $\begin{array}{l}\text { PBG } \\
\text { Cavity } \\
\# 2\end{array}$ & $\begin{array}{l}\text { Pill- } \\
\text { Box } \\
\text { Cavity }\end{array}$ \\
\hline $\begin{array}{ll}\text { Lattice vector } \\
b(\mathrm{~cm})\end{array}$ & \multicolumn{2}{|l|}{0.64} & 0.774 & \\
\hline $\begin{array}{l}\text { Rod radius } a \\
(\mathrm{~cm})\end{array}$ & \multicolumn{2}{|l|}{0.076} & 0.175 & \\
\hline $\begin{array}{l}\text { Cavity radius } \\
(\mathrm{cm})\end{array}$ & \multicolumn{2}{|l|}{2.2} & 2.9 & 0.657 \\
\hline $\begin{array}{l}\text { Frequency } \\
(\mathrm{GHz})\end{array}$ & \multicolumn{2}{|l|}{17.172} & 17.099 & 17.466 \\
\hline Length $L_{\|}(\mathrm{cm})$ & \multicolumn{2}{|l|}{0.45} & 0.45 & 0.45 \\
\hline $\begin{array}{l}\text { Effective } \\
\text { radius } R_{\text {eff }}(\mathrm{cm})\end{array}$ & \multicolumn{2}{|l|}{0.388} & 0.57 & 0.657 \\
\hline $\begin{array}{l}\text { Ohmic } \\
\text { factor } Q_{o h m}\end{array}$ & \multicolumn{2}{|l|}{4200} & 5000 & 5300 \\
\hline $\begin{array}{l}\text { Shunt } \\
\text { impedance } r_{c v} \\
(\mathrm{M} \Omega / \mathrm{cm})\end{array}$ & \multicolumn{2}{|l|}{2.1} & 2.5 & 2.9 \\
\hline $\begin{array}{l}\text { Coupling } \\
\text { scheme }\end{array}$ & Fig.2 & Fig.3 & Fig.4 & Fig.5 \\
\hline $\begin{array}{l}\text { External Q- } \\
\text { factor } Q_{e x t}\end{array}$ & 9000 & 2400 & 9000 & 5500 \\
\hline $\begin{array}{l}\text { Coupling hole } \\
\text { length }(\mathrm{cm})\end{array}$ & & & & 0.2 \\
\hline $\begin{array}{l}\text { Coupling hole } \\
\text { width }(\mathrm{cm})\end{array}$ & & & & 0.37 \\
\hline $\begin{array}{l}\text { Coupling } \\
\text { frequency } \\
(\mathrm{GHz})\end{array}$ & 17.171 & & 17.098 & 17.166 \\
\hline $\begin{array}{l}\text { Maximum } \\
\text { magnetic field } \\
\text { ratio } \\
H_{\max } Z_{d} / E_{\max }\end{array}$ & 1.3 & & 0.95 & 0.94 \\
\hline
\end{tabular}

Table 1. where $\mathrm{E}_{\max }$ is the axial electric field. The results of calculation of the effective radii, Ohmic Q-factors, and shunt impedances are shown in Table 1 for two PBG cavities and a pill-box cavity.

\subsection{External Losses}

We analyze the coupling to the PBG cavity from the WR62 rectangular waveguide (the width of the wide wall is $a_{\mathrm{wg}}=1.575 \mathrm{~cm}$ ). Figure 2 shows the coupling scheme. We excite the cavity from the left waveguide, and the right waveguide is utilized to symmetrize the cavity.

We calculate external losses (an external Q-factor) in two steps using SUPERFISH: (1) determine the resonance frequency of the cavity including the waveguides with the magnetic walls placed on the ends (Fig.2); (2) at this frequency, simulate one cycle of propagation using the initial sinusoidal electric field distribution at the left-side magnetic wall. The ratio of the electric fields, $E_{\max }$ at the axis and $E_{\text {wg }}$ at the left-side wall, gives the external Q-factor:

$$
Q_{\text {ext }}=\left(\frac{E_{\max }}{E_{w g}}\right)^{2} \frac{16 \omega W Z_{w g}}{E_{\max }^{2} a_{w g}}
$$

where $Z_{\mathrm{wg}}=455 \Omega$ is the waveguide impedance.

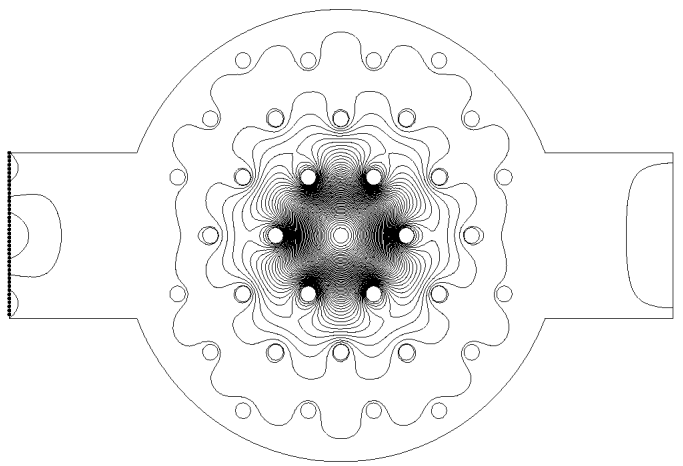

Fig. 2. Cavity \#1, coupling scheme A.

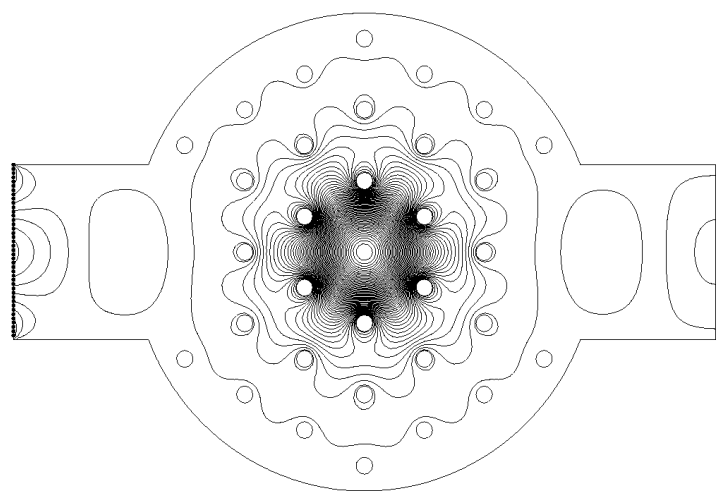

Fig. 3. Cavity \#1, coupling scheme B. 
We treat two schemes of coupling into Cavity \#1: (A) 1 rod from the $3^{\text {rd }}$ row, from both sides, is removed (Fig.2); (B) 2 rods from both sides are removed, and the orientation of the cavity is rotated $90^{\circ}$ (Fig.3). To couple into Cavity \#2, we remove 1 rod of the $3^{\text {rd }}$ row and 1 rod of the $2^{\text {nd }}$ row from both sides (Fig.4). The calculated external Q-factors are shown in Table 1. On the example of Cavity \#1, we indicate a great difference in external Q-factors between the coupling schemes A and B.

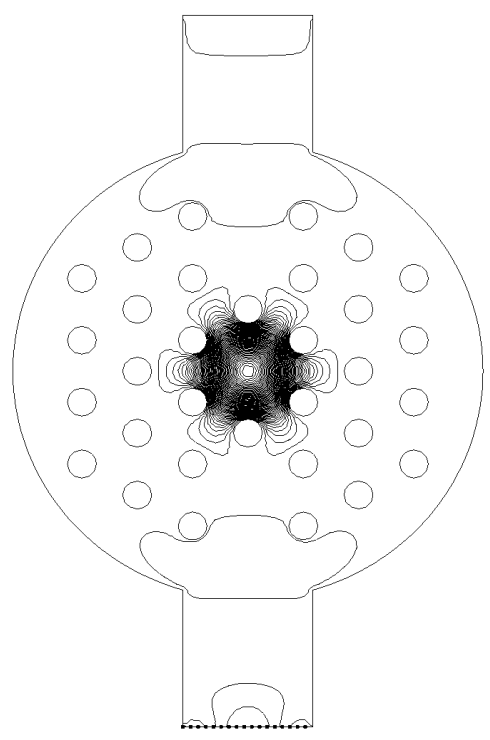

Fig.4. Cavity \#2.

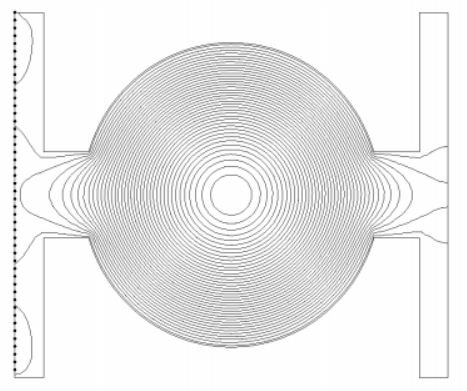

Fig.5. Pill-box cavity.

The pill-box cavity (Fig.5) excitation through the coupling hole is simulated for comparison. The cavity and the coupling hole dimensions are shown in Table 1. Note the relatively small size of the conventional cavity in Fig.5. A critical coupling to the pill-box cavity is obtained since $Q_{\mathrm{ohm}} \approx Q_{\mathrm{ext}}$. Optimization on the rod diameter of the $\mathrm{PBG}$ cavities is required to reach the critical coupling.

The important feature indicated in the PBG cavity is that there is no frequency shift caused by coupling, whereas there is a significant coupling frequency shift in the pill-box cavity (Table 1). This effect is a result of distributed coupling in a PBG cavity. Since the $1^{\text {st }}$ row of rods is not disturbed, the PBG cavity field does not vary due to coupling.

\subsection{Rod Surface Current}

A critical issue is the rod surface current which infers heating while high RF power is coupled into the cavity. The rod surface magnetic field distribution has been calculated, and the maximum magnetic field characterized by its ratio to the axial electric field. This ratio multiplied by $Z_{0}=377 \Omega$ is shown in Table 1 . For the pillbox cavity, we indicate that this ratio reaches its maximum at the coupling hole edge. For the PBG cavity, it is at the inner surface of the rods of the $1^{\text {st }}$ row. The results (Table 1) demonstrate that, for Cavity \#1, the maximum surface magnetic field is about $40 \%$ larger than that for both the pill-box cavity and Cavity \#2.

\section{CONCLUSIONS}

Two examples of $17 \mathrm{GHz}$ PBG accelerating cavities are examined, the resonance frequencies and Q-factors are calculated, and schemes of coupling are designed. We found that coupling into a PBG cavity can be controled by removing the rods from the $3^{\text {rd }}$ row of the lattice. We demonstrated the effect of no frequency shift caused by the coupling, which is a great advantage of PBG cavities. We also demonstrated that the heating of rods in PBG cavities, caused by the magnetic field increase near the rod, is of about the same order of the heating in a coupling-hole unit of a conventional cavity.

\section{REFERENCES}

[1] E.Yablonovitch and T.J.Gmitter, 'Photonic Band Structure: The Face-Centered Cubic Case', Phys. Rev. Lett., 1989, Vol. 63, p. 1950.

[2] J.D.Joannopoulos, R.D.Meade, and J.N.Winn, 'Photonic Crystals: Molding the Flow of Light', Princeton Univ. Press, 1995.

[3] S.Schulz, D.R.Smith, and N.Kroll, 'Photonic Band Gap Resonators for High Energy Accelerators', Proc. of 1993 Particle Accelerator Conference, IEEE, p. 2559.

[4] D.R.Smith, N.Kroll, and S.Schulz, 'Studies of a Metal Photonic Bandgap Cavity', Advanced Accelerator Concepts, AIP Conf. Proc. 335, 1995, p. 761.

[5] D.R.Smith et al., 'Recent Progress on Photonic Band Gap Accelerator Cavities', Advanced Accelerator Concepts, AIP Conf. Proc. 398, 1997, p. 518.

[6] D.R.Smith et al., 'Photonic Band Structure and Defects in One and Two Dimensions', JOSA-B, 1993, Vol.10, p. 314.

[7] S.Trotz et al., 'Experimental Operation of a $17 \mathrm{GHz}$ Photocathode RF Gun', Proc. of 1997 Particle Accelerator Conference, IEEE, p. 2690.

[8] J.Haimson et al., 'A Fully Demountable $550 \mathrm{kV}$ Electron Gun for Low Emittance Beam Experiments with a $17 \mathrm{GHz}$ Linac', Proc. of 1997 Particle Accelerator Conference, IEEE, p. 2808.

[9] J.H.Billen and L.M.Young, 'POISSON/SUPERFISH', LAUR-96-1834, LANL. 